It is not suggested that penicillin alone was responsible for the happy issue. It is well recognized that the immunity responses may so alter that the outcome of such an illness may well transpire to be other than that first envisaged. Nevertheless I am convinced that penicillin in this instance achieved all that was expected of it, whereas in comparable illnesses of previous outbreaks the intensive use of sulphonamides failed to prevent several fatalities. With the probability of further virulent smallpox reaching our ports, it may well fall to others to confirm the impressions gained from the treatment of this patient.

\section{A. Foulis, M.D.}

Senior Assistant Physician and Surgeon, and

Robroyston Auxiliary Hospital Deputy Superintendent. Millerston.

\section{Empyema complicating a Pneumothorax treated with Penicillin}

The following case is interesting in that a pneumothorax occurred as the result of swallowing a safety-pin and a cure was effected by the use of penicillin.

A girl aged 2 years was admitted to hospital on Dec. 17, 1944, having swallowed a safety-pin, which lay point upwards opposite thoracic vertebrae 6 to 8 . The pin was removed by a rotatory movement (in the absence of a pin-closer), and as injury to the oesophagus was suspected the patient was started on a course of sulphathiazole.

A radiograph taken on Dec. 18 showed a right-sided pneumothorax. Nothing except the sulphonamide was given by mouth from Dec. 19 to 22, and the pneumothorax did not increase in size after Dec. 20. The patient now appeared well, but on Dec. 23 there was a dullness at the right base, and a radiograph taken on Dec. 27 showed a small pleural effusion. On Dec. 30 a few c.cm. of thin pus was aspirated, and aspirations were repeated every other day until Jan. 4, 1945, when $50 \mathrm{c.cm}$. of thick pus was obtained. Culture of this pus showed pneumococci, which were penicillin-sensitive. The temperature ranged between $98^{\circ}$ and $100^{\circ}$ until on Jan. 1 it reached $101^{\circ}$. On Jan. 2 sulphathiazole was discontinued.

Penicillin therapy was started on Jan. 4, when 60,000 units in $60 \mathrm{c.cm}$. of normal saline were injected into the right pleural cavity The injection was repeated on Jan. 6 and 8 , and after the third injection the temperature remained normal. $40 \mathrm{c} . \mathrm{cm}$. of thick pus was aspirated on Jan. 11, and a fourth injection was given on that day. This pus and $50 \mathrm{c.cm}$. aspirated on Jan. 17 were sterile, so that the fourth injection may have been unnecessary. Subsequent radiographs showed gradual expansion of the lung but persistent opacity at the right base, which was interpreted as thickened pleura, as no pus was aspirated after Jan. 17.

The patient was discharged on March 17. On March 27, when seen at the out-patient department, she was well, and a radiograph showed complete expansion of the right lung with a small amount of pleural thickening at the parietes.

Hull.

L. A. QUirk, M.D.

\section{Volvulus:- of [Caecum with Reversed Rotation of Mid-gut}

Volvulus of the caecum associated with reversed rotation of the midgut is a rare condition, so that details of the following case may be of interest.

\section{CASE REPORT}

A woman aged 59 was admitted to hospital on Sept. 8, 1943, and tated that twenty-four hours earlier she had begun to have lower abdominal colicky pains, and that her abdomen had become larger. She had vomited several times and had not passed faeces or flatus since the onset of the pain. One week before the present attack she had experienced a similar pain which had lasted for two hours.

On examination the patient was a thin, frail woman in fairly good general condition. The abdomen was distended and tympanitic, the distension being chiefly central abdominal. Audible peristalsis was present and rectal examination revealed no abnormality. A Ryle's tube was passed into the stomach, and the fluid withdrawn was clear and bile-stained. A diagnosis of large-bowel obstruction was made and operation performed.

At operation under a spinal analgesic a right paramedian incision was made and the following condition found: a volvulus of the caecum, first part of the ascending colon, and lower ileum was present, the volvulus consisting of two turns about its axis. The greatly distended caecum was lying in the centre of the abdominal cavity. When the volvulus had been untwisted it was found that the caecum and ascending colon were mobile and had a definite mesentery. The transverse colon iust distal to the hepatic flexure disappeared beneath the mesentery of the small intestine, and reappeared again on the left side. Drainage of the caecum and reappeared again on the left side. Drainage of the caecum ileum, the tube being brought out through a small gridiron incision in the right iliac fossa.

Progress.-The ileostomy tube was removed one week later, and the ileostomy rapidly closed. The patient was discharged from hospital three weeks after operation.

Crumpsall Hospital, Manchester. NORMAN G. Godfrey, F.R.C.S.

\section{Reviews}

\section{A REVOLUTIONARY SCHEME OF LOCAL GOVERNMENT}

Physician, Heal Thyself. A Study of Needs and Means. By G. Scott Williamson, M.D. (Pp. 133. 5s.) London: Faber and Faber.

The title of this book gives little clue to its contents. It is a plea for a revolutionary scheme of local government which Dr. Scott Williamson calls "Liberal Socialism," defined as "the government of society by the individual for the individual." He thinks he can reconcile the claims of the community with those of the individual. He gives a clear and dogmatic exposition of this thesis applied in detail to our medical services. The author is a convinced believer in the rights of the individual, which have been neglected by what he calls " monopoly socialism "-government by bureaucracy. Medical services should be primarily local and directed not centrally but by the residents in the area, who could be trusted, if the means were provided, to see that good medical service would be forthcoming. His experience as medical director of the Peckham Pioneer Clinic has inspired many suggestions which seem reasonable, however Utopian they may appear at first. The overriding duty of the doctor is " to protect his patient," as his " counsel for the defence"; therefore the patient must have his own doctor, and not the State's doctor. Local clinical centres are essential, and all practice must be centred there. They must be "fully equipped with the tools and services required for efficient diagnosis and treatment"; staffed by general practitioners, who are the keystone of the whole service ; the consultants and specialists are advisers used by the doctor in charge of the patient. Great stress is laid on a free transportation service for patients to the centre, to their homes or employment, and to hospital when necessary. So much of the time of doctor and patient might be saved by this as would be equivalent to adding $1 / 2$ or $1 / 3$ doctors to the list, and so enable the new service to start at once. To detect the minor ailments, whose importance he cannot stress too much, all members of the family should be overhauled periodically as at Peckham. These centres must have district hospitals, the beds being under the control of the doctors who send in the patients. "Their own doctor must see them through every illness because only he can assess the success of any treatment." "The sick person is the doctor's patient and the specialist's case." The specialist would be interested, if perhaps annoyed, by what Dr. Williamson has to say about his real status-viz., that of a technical adviser, on certain aspects of a case, to the doctor who is in charge of the patient. The future consultants and teachers should be "super-general practitioners" chosen from among those who have "succeeded in, and not retreated from, general practice." They must be elected by the men among whom they work. They would act as teachers and as executive officers, seeing that the instructions of the patient's own doctor are carried out. Patients must be paid their full wages while sick, their places being taken by apprentices and others who are being trained to do that particular work; trade unions should be responsible for this supply. He holds it to be as logical to pay the workers essential for the community, and to keep their jobs open while sick, as it is to do the same for civil and other servants of the Crown. The centres and hospitals should be under the control of lay committees elected by the potential patients in the area, as being the only people who can say whether the service is satisfactory or not. The role of the central Government is to collect the insurance contributions. It is merely the agent of the individual subscriber, and should no more be allowed to spend his money than his bank would be allowed to spend his savings. Subscriptions to the service should be based not on a flat rate but according to means, and these can be ascertained by dividing the income of the family by the number of dependants. "The rich must not be allowed to contract out of any public service." The local share of the money collected must be handed over to the local committees to spend according to the needs of the locality. The patient will pay his own doctor by drawing a cheque on his "local medical bank." He will no longer be a "panel patient" but "a patient with a cheque book." A suitable way 